DTP-98/71

KCL-MTH/98-40

T-98/106

hep-th/9810098

October 1998

\title{
Generalisations of the Coleman-Thun mechanism and boundary reflection factors
}

\author{
Patrick Dorey円, Roberto Tateo[ and Gérard Watts \\ ${ }^{1}$ Department of Mathematical Sciences, \\ University of Durham, Durham DH1 3LE, England \\ ${ }^{2}$ Service de Physique Théorique, CEA-Saclay, \\ F-91191 Gif-sur-Yvette Cedex, France \\ ${ }^{3}$ Mathematics Department, \\ King's College London, Strand, London WC2R 2LS, U.K.
}

\begin{abstract}
We make a complete pole analysis of the reflection factors of the boundary scaling Lee-Yang model. In the process we uncover a number of previously unremarked mechanisms for the generation of simple poles in boundary reflection factors, which have implications for attempts to close the boundary bootstrap in more general models. We also explain how different boundary conditions can sometimes share the same fundamental reflection factor, by relating the phenomenon to potential ambiguities in the interpretation of certain poles. In the case discussed, this ambiguity can be lifted by specifying the sign of a bulkboundary coupling. While the recipe we employ for the association of poles with general on-shell diagrams is empirically correct, we stress that a justification on the basis of more fundamental principles remains a challenge for future work.
\end{abstract}

\footnotetext{
${ }^{1}$ e-mail: P.E.Dorey@durham.ac.uk

${ }^{2}$ e-mail: Tateo@wasa.saclay.cea.fr

${ }^{3}$ e-mail: gmtw@mth.kcl.ac.uk
} 


\section{Introduction}

The paper by Ghoshal and Zamolodchikov [1] is responsible for a fair amount of the recent work on integrable quantum field theories in the presence of boundaries. The setup they discussed involves a massive model defined on the half line $x \in(-\infty, 0]$, with an integrable boundary condition imposed at $x=0$. The bulk S-matrix must then be supplemented by a boundary S-matrix (a set of so-called reflection factors) describing how each particle bounces off the boundary. This boundary S-matrix is constrained by various consistency conditions - the boundary Yang-Baxter [2], bootstrap [1,3], unitarity and cross-unitarity [1] equations - and one aspect of boundary integrability has been the attempt to match abstract solutions of these constraints with definite boundary conditions for particular models. Even in relatively simple Lagrangian field theories such as the affine Toda models [3 5], this task has proved to be rather hard, a major difficulty being the greatly increased complexity of perturbation theory once spatial translational symmetry has been broken by the presence of the boundary [5 10]. As an alternative line of attack, one might think to check first of all the internal field-theoretic coherence of any proposed boundary S-matrix, before going on to match it with a specific boundary condition. In particular, it is possible to ask whether its poles are telling a sensible story. Ever since the work of Coleman and Thun [11], the rules for playing this game in the bulk have been well established. However, in boundary situations the stock of reliable examples is much more limited, and the situation has remained relatively unclear.

The purpose of this note is to analyse a case which, though simple, seems to exhibit most phenomena one could hope to meet in more complicated situations. This

is the boundary scaling Lee-Yang model, which was studied from various points of view in ref. [12]. A combination of TBA and TCSA techniques, described in [12], allowed the boundary S-matrices to be pinned down unambiguously, and these form the starting-point for the current investigation. We complete the boundary bootstrap, and find 'prosaic' (ie, potentially field-theoretic) explanations for all physical-strip poles. In some situations, physical-strip zeroes of reflection factors have a role to play, providing the boundary analogue of a mechanism previously observed in the bulk behaviour of the non-self-dual affine Toda field theories [13].

Earlier work on the closure of the boundary bootstrap includes the papers by Corrigan et al [5], Fring and Köberle [14, and Saleur and Skorik [15].

\section{The models and their reflection factors}

For a more leisurely discussion of the boundary scaling Lee-Yang models, the reader is referred to ref. [12]; here we just summarise the relevant details. In the bulk, the models are perturbations of the $\mathcal{M}(2 / 5)$ minimal model by its unique relevant spinless field $\varphi$; at the boundary, the possibilities for perturbation depend on which of the two possible conformal boundary conditions is present in the unperturbed 
model. One, denoted $\mathbb{1}$ in [12], does not have any relevant boundary fields, while the other, denoted $\Phi$ in 12], has a single relevant boundary field $\phi$ with scaling dimension $x_{\phi}=-1 / 5$. In the latter case the general perturbed action is thus

$$
\mathcal{A}_{\lambda, \Phi(h)}=\mathcal{A}_{\Phi}+\lambda \int_{-\infty}^{\infty} d y \int_{-\infty}^{0} d x \varphi(x, y)+h \int_{-\infty}^{\infty} d y \phi(y)
$$

where $\mathcal{A}_{\Phi}$ denotes an action for $\mathcal{M}(2 / 5)$ with the $\Phi$ conformal boundary condition imposed at $x=0$, and the bulk and boundary couplings are $\lambda$ and $h$ respectively. The action $\mathcal{A}_{\lambda, \mathbb{1}}$ is similar, but lacks the final term on the right-hand side. For $\lambda>0$ (in the normalisations of [12]) the bulk behaviour is in all cases described by a massive scattering theory with just one particle type, and S-matrix [16]

$$
S(\theta)=-(1)(2) \quad, \quad(x)=\frac{\sinh \left(\frac{\theta}{2}+\frac{i \pi x}{6}\right)}{\sinh \left(\frac{\theta}{2}-\frac{i \pi x}{6}\right)} .
$$

The mass $M$ of this particle is related to the bulk coupling $\lambda$ as $M(\lambda)=\kappa \lambda^{5 / 12}$, where [17] $\kappa=2^{19 / 12} \sqrt{\pi}(\Gamma(3 / 5) \Gamma(4 / 5))^{5 / 12} /\left(5^{5 / 16} \Gamma(2 / 3) \Gamma(5 / 6)\right)$. There is a single $\phi^{3}$-style coupling $f$, seen in the residue of $S(\theta)$ at $\theta=2 \pi i / 3 \equiv i U$, the position of the forward-channel pole:

$$
S(\theta) \simeq \frac{i f^{2}}{\theta-i U}, \quad U=\frac{2 \pi}{3}, \quad f=i 2^{1 / 2} 3^{1 / 4} .
$$

The sign of $f$ is tied to the normalisation of the scattering states, so here we are implicitly settling on one particular choice. At the boundary, reflection factors were proposed and checked against finite-size data in ref. [12]. For the $\mathbb{1}$ and $\Phi(h)$ boundary conditions, they can be written in terms of the blocks $(x)$ defined in (2.2) as

$$
R_{(1)}=\left(\frac{1}{2}\right)\left(\frac{3}{2}\right)\left(\frac{4}{2}\right)^{-1}
$$

and

$$
R_{b}=\left(\frac{1}{2}\right)\left(\frac{3}{2}\right)\left(\frac{4}{2}\right)^{-1}\left(\frac{1-b}{2}\right)^{-1}\left(\frac{1+b}{2}\right)\left(\frac{5-b}{2}\right)\left(\frac{5+b}{2}\right)^{-1}
$$

respectively. The presence of a boundary scale at the $\Phi(h)$ boundary accounts for the dependence of its reflection factor on the parameter $b$, which is related to the bulk and boundary couplings via [12, 18]

$$
h(b)=\sin \left((b+0.5) \frac{\pi}{5}\right) h_{\text {crit }}, \quad h_{\text {crit }}=-\frac{5^{1 / 4} 2^{4 / 5} \pi^{3 / 5} \sin (2 \pi / 5)}{(\Gamma(3 / 5) \Gamma(4 / 5))^{1 / 2}}\left(\frac{\Gamma(2 / 3)}{\Gamma(1 / 6)}\right)^{6 / 5} M(\lambda)^{6 / 5} .
$$




\section{Pole analysis of the basic reflection factors}

The $R_{(1)}$ reflection factor was discussed by Ghoshal and Zamolodchikov [1], albeit without reference to any specific boundary condition. The only details we can add are the previously-mentioned association of $R_{(1)}$ with the $\mathbb{1}$ boundary, and the observation that finite-size data shows no evidence of boundary bound states in this case [12, 19]. This is in accord with the assumptions of ref. [1], where an explanation for the physical-strip poles in $R_{(1)}$ was sought involving just bulk particles and the 'boundary ground state', which we shall label $\mathbb{1}_{0}$. (Later, we shall show that an alternative interpretation of $R_{(1)}$ is also possible, but this is not relevant for the $\mathbb{1}$ boundary condition.) For reflection factors, the physical strip is the region $0 \leq \operatorname{Im} \theta \leq \pi / 2$ of the complex $\theta$-plane, so $R_{(1)}$ has two physical strip poles, one at $i \pi / 6$ and one at $i \pi / 2$. Following [1], both can be explained by postulating the existence of a 'boundary-particle coupling' $g_{\mathbf{1}_{0}}$. The relevant on-shell diagrams are shown in figures la and $1 \mathrm{~b}$.

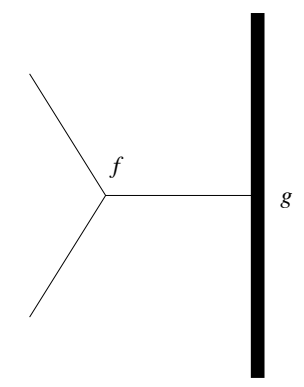

1a) The pole in $R_{(1)}(\theta)$ at $i \pi / 6$

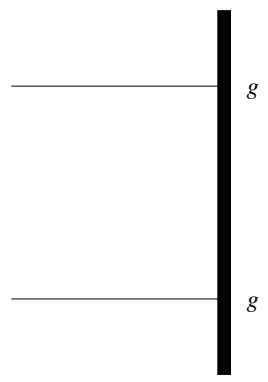

1b) The pole in $R_{(1)}(\theta)$ at $i \pi / 2$

A value can be assigned to the coupling $g_{\mathbf{1}_{0}}$ using one of the rules given in [1]. This determines $g_{\mathbf{1}_{0}}$ through the residue of the pole in $R_{(1)}$ at $\theta=i \pi / 6=i \bar{U} / 2$, where $\bar{U}=\pi-U$ and $U$ is the bulk fusing angle (2.3):

$$
R_{(1)}(\theta) \simeq \frac{i f g_{\mathbf{1}_{0}}}{2 \theta-i \bar{U}}, \quad \bar{U}=\frac{\pi}{3}, \quad g_{\mathbf{1}_{0}}=-i 2 \sqrt{2 \sqrt{3}-3} .
$$

Note that there is no ambiguity about the sign of $g_{\mathbf{1}_{0}}$, once that of $f$ has been fixed. Alternatively, the square of the coupling was related in [1] to the residue at $i \pi / 2$, via $R_{(1)}(\theta) \simeq i\left(g_{\mathbf{1}_{0}}\right)^{2} /(2 \theta-i \pi)$. These can be considered as two definitions of the on-shell coupling, but it must be checked that they are consistent. In fact, this is a consequence of the bootstrap and crossing. To see this, first define, as in ref. [20], $\mathcal{R} F(\theta)$ to be equal to the leading coefficient of the Laurent expansion of the function $F$ about $\theta$ (for simple poles, it is just the residue). In this notation, the first equation in (2.3) becomes $f^{2}=-i \mathcal{R} S(2 \pi i / 3)$, and $\left(g_{\mathbf{1}_{0}}\right)^{2}$ can either be evaluated as 
$-\left(2 \mathcal{R} R_{(1)}(i \pi / 6) / f\right)^{2}$, or as $-2 i \mathcal{R} R_{(1)}(i \pi / 2)$. The equality of these two follows from the identity

$$
\frac{R_{(1)}\left(\frac{i \pi}{6}+\theta\right) R_{(1)}\left(\frac{i \pi}{6}-\theta\right)}{S\left(\frac{2 \pi i}{3}-2 \theta\right)}=R_{(1)}\left(\frac{i \pi}{2}+\theta\right)
$$

which is itself a consequence of the boundary bootstrap equation, together with unitarity, crossing and crossing-unitarity.

The situation for $R_{b}$ is richer. We will initially consider cases where $b$ is real, and can immediately use the symmetries of (2.5) to restrict $b$ to the range $[-3,3]$. Figure 2 shows how the positions of the poles and zeroes of $R_{b}$ (which all lie on the imaginary $\theta$-axis for $b$ real) depend on $b$.

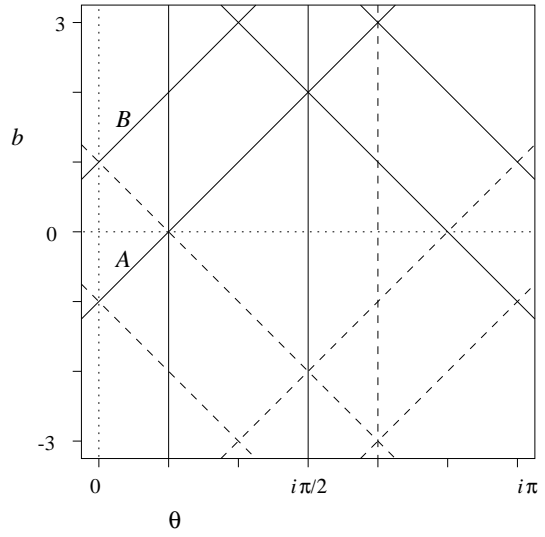

2) Poles and zeroes of $R_{b}(\theta)$. Continuous lines are poles, dashed lines zeroes, and the dotted lines are the $\theta$ and $b$ axes.
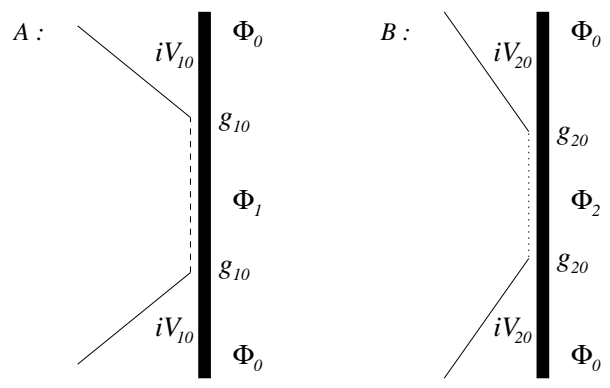

3) The poles $A$ and $B$ in $R_{b}(\theta)$, at $i V_{10}$ and $i V_{20} \cdot \Phi_{1}$ is indicated by a dashed line along the boundary, and $\Phi_{2}$ by a dotted line.

Note that, while the relation (2.6) between $b$ and $h$ has a symmetry about the point $b=2$, the same is clearly not true of the reflection factor $R_{b}$. This issue was discussed in the context of the boundary TBA (BTBA) in ref. [12], where it was found that the BTBA equations rearrange themselves as the point $b=2$ is passed, so that $R_{b}$ is replaced by $R_{4-b}$ and the solutions to the equations are indeed symmetrical about $b=2$. In spite of this fact, it turns out that $R_{b}$ does continue to have an interpretation for $b>2$. As found in ref. 12], the value of the boundary coupling at $b=2$, namely $h_{\text {crit }}$, is the 'critical' value at which the energy of the first excited state (a boundary bound state) becomes degenerate with that of the vacuum. It is therefore natural to expect that continuation through $b=2$ implements a swapping of the vacuum boundary state and the first boundary bound state, and it will be seen shortly that this is precisely what happens at the level of the reflection factors, with $R_{b>2}$ becoming the reflection factor for the first boundary bound state. 
First, though, we will treat the pole structure while $b$ remains in the range $[-3,2]$. Apart from the exceptional points $b= \pm 2$, there are always simple poles at $\theta=i \pi / 6$ and $\theta=i \pi / 2$. These are explained, just as previously, by postulating a boundaryparticle coupling $g_{\Phi_{0}}$ between the bulk particle and $\Phi_{0}$, the boundary ground state for the $\Phi(h)$ boundary condition. For brevity, we will write this coupling as $g_{0}$. Its value depends on $h$, or equivalently on $b$, in the following way:

$$
g_{0}(h(b))=\frac{\tan ((b+2) \pi / 12)}{\tan ((b-2) \pi / 12)} g_{\mathbf{1}_{0}} .
$$

The sequence of arguments culminating in equation (3.2) applies here essentially unchanged, and so there is no need to make a separate check of the compatibility of the residues at $i \pi / 6$ and $i \pi / 2$. (A small subtlety arises when $b=0$, but this will be described later.)

For $b \in[-3,-1]$, this is the end of the story. Then as $b$ passes -1 , an extra pole enters the physical strip, at $i \pi(b+1) / 6$; and as $b$ passes 1 , a second appears, at $i \pi(b-1) / 6$. These $b$-dependent poles are labelled $A$ and $B$ in figure 2 . It is natural to associate them with boundary bound states, and an analysis of finite-size data confirms this [12]. Writing the two states as $\Phi_{1}$ and $\Phi_{2}$, the situation can be formalised by introducing a couple of further boundary-particle couplings $g_{10}$ and $g_{20}$, with corresponding 'boundary fusing angles' $V_{10}=(b+1) \pi / 6$ and $V_{20}=(b-1) \pi / 6$, as depicted in figure 3 . The energies $e_{1}$ and $e_{2}$ of the boundary bound states are given by $e_{j}-e_{0}=M \cos \left(V_{j 0}\right)$, where $e_{0}$ is the energy of $\Phi_{0}$. (As mentioned above, $e_{1}-e_{0}=0$ at $b=2$.) Finally, the reflection factors $R_{b}^{[1]}$ and $R_{b}^{[2]}$ for a particle bouncing off the two boundary bound states can be computed, using the 'boundary bound-state bootstrap equation' [1]:

$$
R_{b}^{[j]}(\theta)=S\left(\theta-i V_{j 0}\right) S\left(\theta+i V_{j 0}\right) R_{b}(\theta), \quad j=1,2 .
$$

This yields

$$
\begin{aligned}
& R_{b}^{[1]}=\left(\frac{1}{2}\right)\left(\frac{3}{2}\right)\left(\frac{4}{2}\right)^{-1}\left(\frac{1+b}{2}\right)\left(\frac{3-b}{2}\right)\left(\frac{3+b}{2}\right)\left(\frac{5-b}{2}\right), \\
& R_{b}^{[2]}=\left(\frac{1}{2}\right)\left(\frac{3}{2}\right)\left(\frac{4}{2}\right)^{-1}\left(\frac{1-b}{2}\right)^{-1}\left(\frac{1+b}{2}\right)^{2}\left(\frac{3-b}{2}\right)\left(\frac{3+b}{2}\right)\left(\frac{5-b}{2}\right)^{2}\left(\frac{5+b}{2}\right)^{-1} .
\end{aligned}
$$

The exchange of the vacuum and the first boundary bound state as $b$ passes through 2 can now be seen in the equality $R_{4-b}^{[1]}(\theta)=R_{b}(\theta)$, whilst the symmetry $R_{4-b}^{[2]}(\theta)=$ $R_{b}^{[2]}(\theta)$ reflects the absence of any such exchange at $b=2$ involving the second boundary bound state. The functions $R_{b}^{[1]}$ and $R_{b}^{[2]}$ are shown in figures 4 and 5 , and the next section is concerned with their pole structures. 


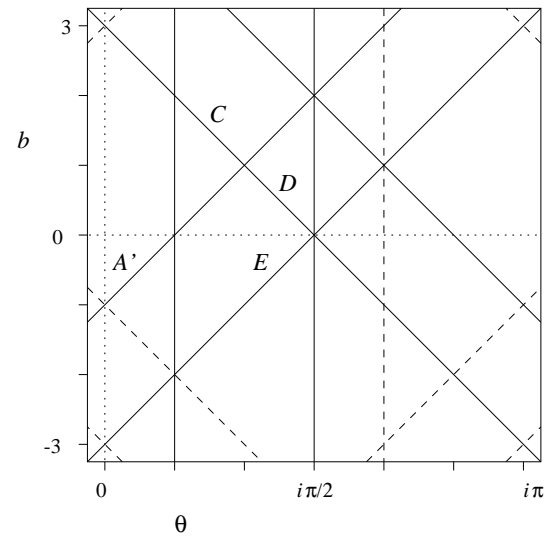

4) Poles and zeroes of $R_{b}^{[1]}(\theta)$. Notation as in figure 2 .

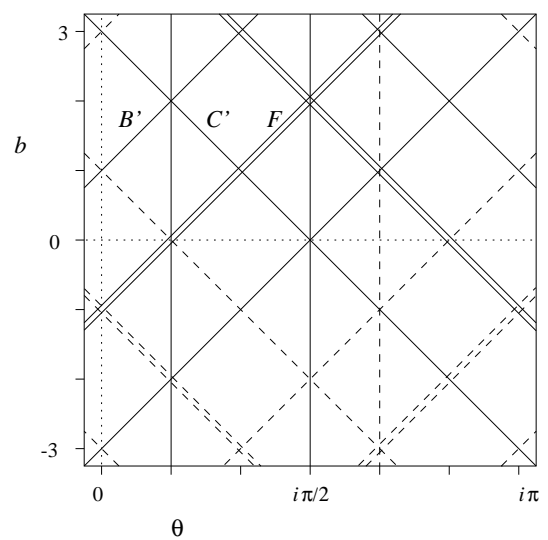

5) Poles and zeroes of $R_{b}^{[2]}(\theta)$. Notation as in figure 2 , with double poles and zeroes indicated by double lines.

\section{Pole analysis of the higher reflection factors}

The treatment of the higher reflection factors can be confined to those ranges of $b$ for which the relevant boundary bound state is present in the spectrum of the model. For $R_{b}^{[1]}$ this is the range $[-1,2]$, and for $R_{b}^{[2]}$, the range $[1,2]$. The poles in both reflection factors at $i \pi / 6$ and $i \pi / 2$ are dealt with as before, via diagrams of the sort shown in figure 1.

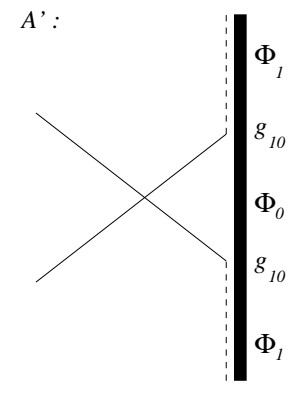

6) The poles $A^{\prime}$ in $R_{b}^{[1]}(\theta)$ and $B^{\prime}$ in $R_{b}^{[2]}(\theta)$, at $i V_{10}$ and $i V_{20}$ respectively. Notation and fusing angles as in figure 3 .
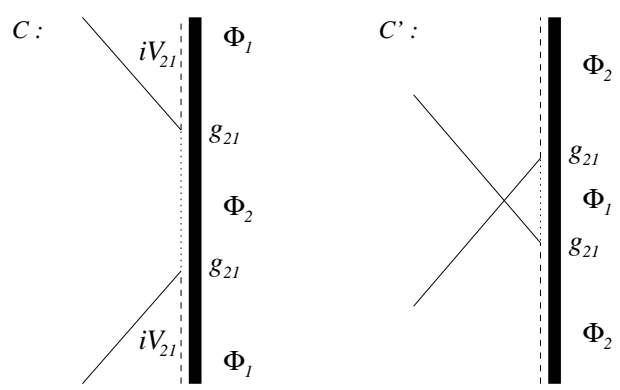

Next, consider the pole in $R_{b}^{[1]}$ at $i V_{10}$, labelled $A^{\prime}$ in figure 4 . This occurs at the same place as the pole in the basic reflection factor $R_{b}$ which gave rise to $R_{b}^{[1]}$ in 
the first place. Its existence can be traced to the first on-shell diagram of figure 6, the boundary analogue of a $u$-channel diagram in the bulk. An identical mechanism accounts for the pole $B^{\prime}$ in $R_{b}^{[2]}$, and the relevant on-shell diagram is also shown.

This ' $u$-channel' mechanism is a generic feature of higher reflection factors, and does not seem to have been remarked before. In particular, in [5] an infinite tower of boundary states was suggested for a certain boundary condition in the $a_{2}^{(1)}$ affine Toda theory, based on the assumption that such poles always correspond to new boundary bound states. While this cannot be absolutely ruled out in the Toda context, it would now appear to be an unnecessarily complicated scenario. Similar poles were also discussed in the boundary sine-Gordon model in 15], where they were rejected on the basis of a Bethe ansatz solution of a related lattice model. However, an interpretation within the continuum boundary field theory was not given in that paper.

When $b$ is in the range $[1,2]$, there is also the possibility to form the second boundary bound state as an excited state of the first. Indeed, both $R_{b}^{[1]}$ and $R_{b}^{[2]}$ have simple poles at $\theta=i V_{21}$, where $V_{21}=(3-b) \pi / 6$. These are labelled $C$ and $C^{\prime}$ on figures 4 and 5 , and the equality

$$
e_{2}=e_{1}+M \cos V_{21}
$$

shows that their positions match such an interpretation. We therefore deduce that the coupling $g_{21}$ is nonzero whenever both $\Phi_{1}$ and $\Phi_{2}$ are in the spectrum. The relevant on-shell diagrams are shown in figure 7 .

The remaining poles are more delicate. Consider first the continuation of the pole $C$ below $b=1$, labelled $D$ on figure 4 . Since $\Phi_{2}$ is not in the spectrum for $b<1$, figure 7 can no longer be invoked to explain this pole. However, precisely as $b$ drops below 1 , it becomes geometrically possible to draw a new on-shell diagram, as in figure 8 .

The quoted angles follow from the on-shell conditions at each three-point vertex. The rapidity of the incoming particle is $i\left(2 \pi / 3-V_{20}\right)=i(3-b) \pi / 6=i V_{21}$, which is indeed the location of the pole $D$. However the most straightforward application of the rule found in bulk scattering would suggest that the diagram be associated with a second-order pole, rather than the first order required for a match with the exact reflection factor. This can be resolved by the same generalisation of the ColemanThun mechanism as previously found to operate in the bulk S-matrices of non selfdual affine Toda field theories [13]. Observe that the predicted higher pole should be multiplied by a collection of vertex factors, and for the process under discussion these comprise not only the three-point vertices $f^{2} g_{\mathbf{1}_{0}}^{2}$, but also a ground-state reflection factor $R_{b}(\theta)$, evaluated at $\theta=i\left(\pi / 3-V_{10}\right)=i(1-b) \pi / 6$. But from (2.5) or figure 2 , $R_{b}(\theta)$ has a zero at exactly this point. Thus the prediction is demoted to first order, as required.

The remaining physical-strip poles are dealt with similarly. For $b$ in the range 


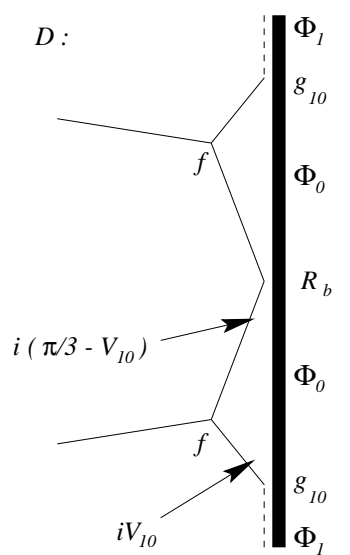

8) The pole $D$ in $R_{b}^{[1]}(\theta)$ at $i\left(2 \pi / 3-V_{20}\right)$, present for $b \in[0,1]$.

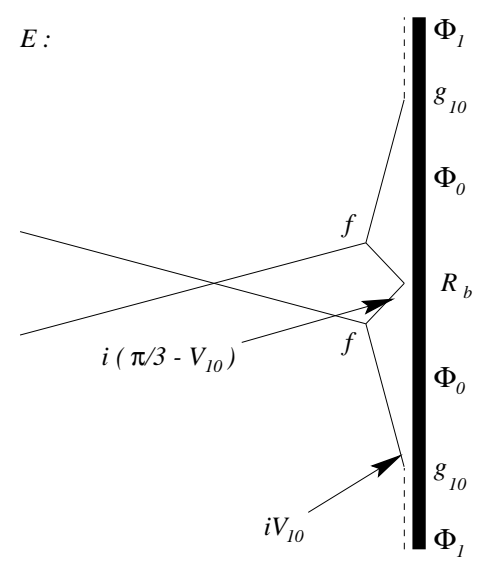

9) The pole $E$ in $R_{b}^{[1]}(\theta)$ at $i\left(\pi / 3+V_{21}\right)$, present for $b \in[-1,0]$.

$[-1,0]$, figure 8 can no longer be drawn, but in its place the diagram drawn as figure 9 becomes possible. As before, a generalised Coleman-Thun mechanism results in a first-order pole, correctly-placed to match the pole marked $E$ on figure 4 . Then $R_{b}^{[2]}(\theta)$ has a double pole at $i(b+1) \pi / 6$, marked $F$ on figure 5 . We leave it to the reader to check that two different diagrams contribute. The first is similar to figure 8 , but with the sequence of boundary states down the right-hand side replaced by $\left\{\Phi_{2}, \Phi_{1}, \Phi_{1}, \Phi_{2}\right\}$, while the second has the form of figure 9 , with the sequence $\left\{\Phi_{2}, \Phi_{0}, \Phi_{0}, \Phi_{2}\right\}$. In both the 'internal' reflection factor $\left(R_{b}^{[1]}\right.$ or $R_{b}$ respectively) does not this time have a zero at the relevant point, so the pole remains secondorder. Finally, there are various isolated values of $b$ at which higher-order poles appear. It is straightforward to check that these also have explanations in terms of 'exceptional' on-shell diagrams, which can only be drawn at these specific points.

\section{Conclusions}

One perhaps unexpected bonus of the analysis just completed is that it enables us to resolve a puzzle that was mentioned in the concluding section of [12]. There it was noted that $R_{b=0}$, the reflection factor for the $\Phi(h(0))$ boundary, is exactly the same $R_{(1)}$, the reflection factor for the $\mathbb{1}$ boundary. This is in spite of the fact that the physics of the two boundary conditions, as seen, for example, in finite-size spectra, are very different. We now see that, while the functions are the same, the pole structures have different interpretations in the two cases. For $R_{(1)}(\theta)$, the pole at $i \pi / 6$ can be traced back to the on-shell diagram of figure 1a, and a boundaryparticle coupling for the $\mathbb{1}$ boundary equal to $g_{\mathbf{1}_{0}}$. By contrast, equation (3.3) taken 
at $b=0$ reveals that the corresponding coupling for the $\Phi(h(0))$ boundary, namely $g_{0}(h(0))$, is equal to $-g_{\mathbf{1}_{0}}$. Thus the contribution of the diagram of figure 1a in this case is exactly the negative of that required for a match with the reflection factor. Agreement is restored by the fact that the residue at $i \pi / 6$ picks up an exceptional extra contribution from the formation of the $\Phi_{1}$ boundary bound state, the left hand diagram of figure 3. Thus there are two consistent interpretations of the $R_{(1)}$ reflection factor, depending on the sign given to the bulk-boundary coupling. The 'wrong' sign forces the introduction of a boundary bound state to correct the residue at $i \pi / 6$, but the bootstrap can then be consistently closed on this extra state. A complete set of infrared data in this situation therefore comprises not only the bulk S-matrix and the elementary boundary reflection factor, but also the sign of the bulk-boundary coupling. This sign turns out to have an important role to play in the calculation of correlation functions through the form-factor approach, but we will leave a detailed discussion of this point to another paper.

The restriction to real values of $b$ has confined the boundary field $h$ to the range $\left[-\left|h_{\text {crit }}\right|,\left|h_{\text {crit }}\right|\right]$. What happens at other real values of $h$ ? For $h>\left|h_{\text {crit }}\right|$, all seems to be well. Examining the formula (2.6), we simply have to set $b=-3+i \vec{b}$, and continue away from $b=-3$ through real values $\widehat{b}$. For such complex values of $b, R_{b}(\theta)$ is still real-analytic. It has poles at $i \pi / 6$ and $i \pi / 2$ which can be explained just as before, and a pair of physical-strip zeroes at $i \pi / 3 \pm \widehat{b} \pi / 6$. These (or, better, their accompanying poles on the unphysical sheet) might perhaps have an interpretation in terms of unstable resonances, but in any case they are harmless as far as the properties of the boundary reflection factor are concerned. By contrast, for $h<-\left|h_{\text {crit }}\right|$, the picture is less promising. The continuation of (2.6) requires us to set $b=2+i \widehat{b}$, resulting in a reflection factor $R_{b}$ which is no longer real analytic. In fact, such behaviour is only to be expected, given the observation of [12 that values of the boundary field $h$ less than $-\left|h_{\text {crit }}\right|$ destabilise the bulk vacuum.

This completes the pole analysis of the model at all values of the boundary field. We can claim to have employed a coherent set of rules, which has allowed us to rederive a pole structure and spectrum that we knew independently (from BTCSA results) to be correct. This should be useful in other contexts where BTCSA results are not yet available. In particular it is possible to resolve the discrepancies between boundary bootstrap and lattice Bethe ansatz results for the boundary sine-Gordon model that were reported in 15. One aspect of this, the $u$-channel pole mechanism, was mentioned above; a more complete analysis will appear elsewhere.

Another interesting feature revealed by the above analysis and our previous paper [12] is the way that the BTBA equations rearrange themselves as the point $b=2$ is passed so as to continue to yield the correct ground state energy, even though the reflection factors of the ground and first excited states themselves swap over. Again, we expect such phenomena to reappear in more complicated models.

While the rules employed here have proved to be reliable, we should reiterate that they have been obtained merely as the natural generalisation of the bulk situation, 
rather than from any fundamental principles. Even to verify some higher poles perturbatively would be reassuring, and if the bulk 21] is anything to go by, the affine Toda field theories may provide the most tractable examples. This might appear to be a distant goal, given the complexities of the most basic perturbative calculations in boundary models. However the situation might simplify when attention is restricted to a check of pole residues, and the possibility should certainly be explored.

Acknowledgements - We would like to thank Andrew Pocklington and Robert Weston for discussions on related issues. The work was supported in part by a TMR grant of the European Commission, contract reference ERBFMRXCT960012, and in part by an EPSRC grant GR/K30667. PED and GMTW thank the organisers of the Oberwolfach workshop on integrable systems for hospitality and the EPSRC for Advanced Fellowships, and RT thanks SPhT Saclay for hospitality.

\section{References}

[1] S. Ghoshal and A.B. Zamolodchikov, 'Boundary $S$ matrix and boundary state in two-dimensional integrable quantum field theory', Int. J. Mod. Phys. A9 (1994) 3841, hep-th/9306002.

[2] I.V. Cherednik, 'Factorizing particles on a half-line and root systems', Teor. Math. Phys. 61 (1984) 977.

[3] A. Fring and R. Köberle, 'Factorized scattering in the presence of reflecting boundaries', Nucl. Phys. B421 (1994) 159, hep-th/9304141.

[4] R. Sasaki, 'Reflection bootstrap relations for Toda field theory', Plenary talk at the International Conference on Interface between Physics and Mathematics IPM93, Hangzhou, China; YITP/U-93-33, hep-th/9311027.

[5] E. Corrigan, P.E. Dorey, R.H. Rietdijk and R. Sasaki, 'Affine Toda field theory on a half-line', Phys. Lett. B333 (1994) 83-91, hep-th/9404108.

[6] E. Corrigan, P.E. Dorey and R.H. Rietdijk, 'Aspects of affine Toda field theory on a half line', Prog. Theor. Phys. Suppl. 118 (1995) 143-164, hep-th/9407148.

[7] J.D. Kim, 'Boundary reflection matrix in perturbative quantum field theory', Phys. Lett. B353 (1995) 213, hep-th/9504018.

[8] E. Corrigan, 'On duality and reflection factors for the sinh-Gordon model', Int. J. Mod. Phys. A13 (1998) 2709-2722, hep-th/9707235.

[9] N. Topor, 'Perturbation method for boundary $S$-matrix in 2D quantum field theory', Mod. Phys. Lett. A12 (1997) 2951-2962. 
[10] M. Perkins and P. Bowcock, 'Quantum corrections to the classical reflection factor in $a_{2}^{(1)}$ Toda field theory', Durham preprint DTP 98-49, hep-th/9807146.

[11] S. Coleman and H.J. Thun, 'On the prosaic origin of the double poles in the sine-Gordon S-matrix', Commun. Math. Phys. 61 31-39.

[12] P. Dorey, A. Pocklington, R. Tateo and G.M.T. Watts, 'TBA and TCSA with boundaries and excited states', Nucl. Phys. B525 (1998) 641-663, hep-th/9712197.

[13] E. Corrigan, P.E. Dorey and R. Sasaki, 'On a generalised bootstrap principle', Nucl. Phys. B408 (1993) 579-599, hep-th/9304065.

[14] A. Fring and R. Köberle, 'Boundary bound states in affine Toda field theory' Int. J. Mod. Phys. A10 (1995) 739-752, hep-th/9404188.

[15] H. Saleur and S. Skorik, 'Boundary bound states and boundary bootstrap in the sine-Gordon model with Dirichlet boundary conditions', J. Phys. A28 (1995) 6605-6622, hep-th/9502011.

[16] J.L. Cardy and G. Mussardo, ' $S$ matrix of the Yang-Lee edge singularity in two-dimensions', Phys. Lett. B225 (1989) 275.

[17] Al.B. Zamolodchikov, 'Mass scale in sine-Gordon model and its reductions', Int. J. Mod. Phys. A10 (1995) 1125.

[18] P. Dorey, I. Runkel, R. Tateo and G.M.T. Watts, ' $g$-function flow in perturbed boundary conformal field theories', to appear.

[19] P. Dorey, I. Runkel, R. Tateo and G.M.T. Watts, 'TBA and TCSA with boundaries and excited states II', to appear.

[20] P. Dorey and R. Tateo, 'Excited states in some simple perturbed conformal field theories', Nucl. Phys. B515 (1998) 575, hep-th/9706140.

[21] H.W. Braden, E. Corrigan, P.E. Dorey and R. Sasaki, 'Multiple poles and other features of affine Toda field theory' Nucl. Phys. B356 (1991) 469-498. 